Prof. Dr. Peter Berlit

Deutsche Gesellschaft für Neurologie (DGN)

Reinhardtstr. 27C, 10117 Berlin, Deutschland

berlit@dgn.org

\title{
Schlaganfallversorgung in Deutschland - gemeinsam sind wir stark!
}

Eine der großen Erfolge der Neurologie in Deutschland ist die europa-, ja vielleicht sogar weltweit vorbildliche flächendeckende Schlaganfallversorgung. Die Einführung des Stroke-Unit-Konzepts im Jahr 1994 führte zu einer messbaren Reduktion von Mortalität und Pflegebedürftigkeit. Der auf den Spezialstationen betriebene apparative und personelle Aufwand wird über spezielle OPS-Ziffern (OPS: Operationen- und Prozedurenschlüssel) gegenfinanziert. Vor mehr als 10 Jahren wurden für die seltenen Fälle raumfordernder Infarkte mit der Notwendigkeit zur Hemikraniektomie Verlegungskriterien entwickelt. Bei Fehlen einer entsprechenden Abteilung im Hause sollte ein Kooperationspartner in höchstens halbstündiger Transportentfernung „unter Verwendung des schnellstmöglichen Transportmittels (z. B. Hubschrauber)“ erreichbar sein. Der 1. Senat des Bundessozialgerichts erkannte am 19.06.2018 für Recht: „Die höchstens halbstündige Transportentfernung bemisst sich nach dem Zeitintervall zwischen Rettungstransportbeginn, dem Ingangsetzen der Rettungskette durch die Entscheidung, ein Transportmittel anzufordern, und Rettungstransportende, der Übergabe des Patienten an die behandelnde Einheit im Kooperationspartner-Krankenhaus. “ Aufgrund dieses Urteils stellten Kostenträger die Zahlung der unter der OPS-Ziffern 8-981 und 8-98b abgerechneten Fälle für 4 Jahre unter den Vorbehalt der nachträglichen Rückforderung. Alle Häuser ohne eigene Neurochirurgie sowie interventionellneuroradiologische oder gefäßchirurgische Versorgungsmöglichkeit wurden zur Darstellung der Strukturgegebenheiten der Schlaganfallkomplexbehandlung aufgefordert. Die möglichen Rückforderungen der Krankenkassen in 3-stelliger Millionenhöhe führten zu einer akuten Gefährdung der wirtschaftlichen Existenz zahlreicher neurologischer Versorgungseinrichtungen.

\section{Offener Brief an den Bundesgesundheitsminister}

In einer vorbildlichen Weise reagierten DGN (Deutsche Gesellschaft für Neurologie), BDN (Berufsverband Deutscher Neurologen) und DSG (Deutsche Schlaganfall-Gesellschaft) sofort

DGNeurologie 2018 • 1 (2): 77-78

https://doi.org/10.1007/s42451-018-0032-4

๑) Springer Medizin Verlag GmbH, ein Teil von Springer Nature 2018 gemeinsam, indem sie in einem Brief an den Bundesgesundheitsminister und die gesundheitspolitischen Sprecher der Fraktionen die Auswirkungen des BSG-Urteils auf die Schlaganfallversorgung darstellten und ein politisches Eingreifen forderten. Parallel erfolgten juristische Beratungen und persönliche Gespräche, u. a. mit dem Spitzenverband der Krankenkassen.

Das Deutsche Institut für Medizinische Dokumentation und Information (DIMDI), eine Behörde im Geschäftsbereich des Bundesministeriums für Gesundheit (BMG), stellte im August 2018 den Text für die Kodes aus den Bereichen 8-981 und 8-98b klar: „Die Zeit zwischen Rettungstransportbeginn und Rettungstransportende ist die Zeit, die der Patient im Transportmittel verbringt".

Der Bundesrat bat am 21.09.2018 die Bundesregierung, „Maßnahmen zu prüfen, die eine qualitativ hochwertige Schlaganfallversorgung auch in den Flächenländern weiterhin nachhaltig sicherstellen".

Hierzu stellte die Bundesregierung fest: „Die bei der neurologischen Komplexbehandlung des akuten Schlaganfalls maßgebliche Transportzeit wird im Operationen- und Prozedurenschlüssel für das Jahr 2019 dahingehend präzisiert, dass es auf die Zeit ankommt, die der Patient oder die Patientin im Transportmittel verbringt. Abweichende Auslegungen, die auf den Zeitraum ab der Feststellung einer Verlegungsnotwendigkeit bis zu einem möglichen Behandlungsbeginn abzielen und die nach der Einschätzung des Bundesrates zu einer Gefährdung der flächendeckenden, qualitativ hochwertigen Schlaganfallversorgung führen können, haben damit im Wortlaut des einschlägigen Operationen- und Prozedurenschlüssels keine Grundlage mehr. Darüber hinaus wird geprüft, ob und inwieweit ergänzende gesetzliche Regelungen erforderlich sind. $\$ 301$ Abs. 2 SGB V wird dahingehend ergänzt, dass das Deutsche Institut für medizinische Dokumentation und Information bei Auslegungsfragen zu den Diagnosen- und Prozedurenschlüsseln Klarstellungen mit Wirkung auch für die Vergangenheit vornehmen kann. Mit dieser Regelung möchte das BMG künftig vermeiden, dass aufgrund von Auslegungsunsicherheiten eine Vielzahl von zurückliegenden Abrechnungsverfahren erneut aufgegriffen und abgerechnet werden“. Diese Klarstellungen helfen uns schon deutlich weiter. Entscheidend für die Zukunft ist jedoch die Schaffung einer rechtssicheren Formulierung für die Verlegungssituation; wir Neurologen wissen, dass es dabei ja weniger um neurochir- 
urgische Eingriffe als um neuroradiologisch-interventionell indizierte Verlegungen geht.

Die OPS-Ziffern stammen von 2007, die Thrombektomie ist aber erst seit 2016 eine etablierte Methode! Natürlich sind alle evidenzbasierten Therapieverfahren der akuten Schlaganfalltherapie bis zu einem gewissen Grad zeitkritisch. Aber Kraniotomien können vorausschauend geplant werden. Und die etwa 5-8 \% aller Schlaganfälle, die für eine Thrombektomie in Frage kommen, machen eine fundierte Vorselektion durch qualifizierte Diagnostik erforderlich. Eine Umleitung aller Schlaganfallpatienten aus wohnortnahen Stroke-Units in überregionale Versorgungszentren würde diese Menschen durch längere Transportzeiten und später einsetzende Therapie (systemische Thrombolyse) unnötig benachteiligen. Eine Studie zur Logistik mit unterschiedlichen Zeit- und Modellkalkulationen ergab, dass eine Transportzeit („travel time“) von 60 min als Toleranzgrenze („cut off“) für eine effektive Logistik vertretbar wäre [1].

Wir werden als Fachgesellschaft weiter aktiv gemeinsam mit der Schlaganfallgesellschaft und dem Berufsverband die wissenschaftlichen Kenntnisse in praxistaugliche Konzepte umsetzen - im Interesse unserer vorbildlichen Schlaganfallversorgung in Deutschland. Wenn Sie dazu Anregungen haben, schreiben Sie mir!

Ihr

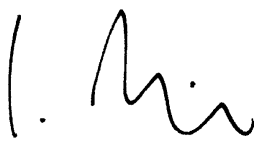

Peter Berlit

Literatur

1. Holodinsky JK et al (2018) Modeling stroke patient transport for all patients with suspected large-vessel occlusion. Jama Neurol. https://doi.org/10.1001/jamaneurol.2018.2424

\section{Ausschreibung}

\section{Robert-Wartenberg-Preis 2019}

Bewerbungsfrist: 15. Dezember 2018

Dotation: 5.000 Euro

Die Kommission der leitenden Krankenhausärzte schreibt die RobertWartenberg-Lecture aus. Der Preis richtet sich an wissenschaftlich, jedoch nicht an universitären Einrichtungen tätige Neurologen (Klinikärzte: primär Oberärzte, Fachärzte, Assistenzärzte), deren Arbeiten Bezug zur klinischen Neurologie haben und konkret Anwendung finden können. Der Preis ist mit einer Vorlesung auf der Jahrestagung der Leitenden Krankenhausärzte in Kassel am 8. März 2019 verbunden und wird in Form einer Urkunde überreicht und ist mit einer Geldprämie von 5.000 Euro verbunden.

\section{Preiskuratorium}

Das Preiskuratorium setzt sich aus der Präsidentin der DGN und den Mitgliedern der Kommission "Leitende Krankenhausärzte" zusammen.

\section{Bewerbung}

Es soll eine bereits veröffentlichte, eine zur Veröffentlichung angenommene wissenschaftliche Einzelarbeit oder ein für den Bewerber repräsentatives wissenschaftliches Werk (an einer nichtuniversitären Einrichtung entstanden oder dort fortgesetzt) zusammen mit einem Curriculum vitae und einem Schriftenverzeichnis eingereicht werden. Die Unterlagen senden Sie bitte per Post und zusätzlich als PDF per E-Mail bis spätestens 15. Dezember 2018 an:

Prof. Dr. Karl Wessel

Neurologische Klinik

Klinikum Braunschweig

Salzdahlumer Str. 90

38126 Braunschweig

k.wessel@klinikum-braunschweig.de

Quelle: Deutsche Gesellschaft für Neurologie, www.dgn.de 